\title{
Incidencia de la última remuneración en la liquidación por terminación del contrato de trabajo
}

\section{Impact of the last remuneration on the settlement for termination of the employment contract}

\author{
Yanhet Lucia Valverde-Torres \\ ds.luisdsm44@uniandes.edu.ec \\ Universidad Regional Autónoma de los Andes, Santo Domingo \\ Ecuador \\ https://orcid.org/0000-0002-0891-286X \\ Luis David Salinas-Mirava \\ ds.luisdsm44@uniandes.edu.ec \\ Universidad Regional Autónoma de los Andes, Santo Domingo \\ Ecuador \\ https://orcid.org/0000-0001-6749-5309
}

Recepción: 15 de septiembre 2021

Revisado: 25 octubre 2021

Aprobación: 15 de noviembre 2021

Publicación: 01 de diciembre 2021 


\title{
RESUMEN
}

En la presente investigación se planteó como objetivo general analizar jurídicamente la Incidencia de la última remuneración en la liquidación por terminación del contrato de trabajo en Ecuador. La metodología utilizada desde la perspectiva cualitativa, se apoyó con el método de investigación el analítico-sintético, ya que mediante el análisis que se realizó se descompuso el tema investigado en sus partes y cualidades más relevantes. Asimismo, se utilizó el análisis documental con la finalidad de adquirir información sobre los derechos laborales. Se realizaron entrevistas a abogados conocedores de la materia laboral y a jueces laborales en la ciudad de Santo Domingo para que desde su experiencia manifestaran como en las indemnizaciones de trabajadores con remuneraciones con ingresos variables pueden causar vulneraciones reconocido en la Constitución y normas. Se concluye, que no se puede hablar de que exista una vulneración, aunque si podría existir una mala aplicación a favor del empleador.

Descriptores: Remuneración; indemnización; trabajo; derecho laboral; ley. (Palabras tomadas de Tesauro UNESCO).

\begin{abstract}
The general objective of this research was to legally analyze the incidence of the last remuneration in the settlement for termination of the employment contract in Ecuador. The methodology used from the qualitative perspective was supported by the analytical-synthetic research method, since through the analysis that was carried out, the investigated topic was decomposed into its most relevant parts and qualities. Likewise, documentary analysis was used in order to acquire information on labor rights. Interviews were conducted with lawyers familiar with labor matters and with labor judges in the city of Santo Domingo so that, from their experience, they could demonstrate how compensation for workers with variable earnings can cause violations recognized in the Constitution and regulations. It is concluded that it cannot be said that there is a violation, although there could be a bad application in favor of the employer.
\end{abstract}

Descriptors: Remuneration; compensation; job; labor law; law. (words takenfrom UNESCO Thesaurus). 


\section{INTRODUCCIÓN}

El contrato individual de trabajo, es el convenio en el cual un individuo se compromete para con otra a prestar sus servicios lícitos y personales, bajo su dependencia, por una remuneración fijada por el convenio de ley (Código del Trabajo, 2005).

El artículo 11 del Código de Trabajo (2005) menciona la siguiente clasificación:

a) Expreso o tácito, escrito o verbal;

b) A sueldo, a jornada, en participación y mixto;

c) Tiempo indefinido, temporada, eventual y ocasional

d) Por obra cierta, por obra o servicio determinado dentro del negocio, por tarea o a destajo; e

e) Individual, grupo o equipo.

Así mismo, en su artículo 21, los elementos o requisitos de un contrato escrito que esencialmente debe tener es la clase de trabajo objeto del contrato; la forma en cómo se va a ejecutar. por unidades de tiempo u obra, por tarea; forma de pago; tiempo de duración del contrato, lugar donde se ejecuta la obra o el trabajo; las declaraciones si se establece o no las sanciones, en el caso de serlo determinarlas y las garantías para su efectividad.

El trabajo es un derecho constitucional en virtud de lo establecido en la Constitución de la República (2008) en su artículo 33, menciona al trabajo como un derecho y una responsabilidad social, con un fondo económico, la cual es fuente de vida digna y la base angular de la superación personal. El Estado debe respetar y hacer respetar el derecho a la dignidad humana, mediante el establecimiento de políticas que plasmen este fin. Garantiza además el derecho a un trabajo saludable y libremente escogido o aceptado. Por otro lado, Masabanda Analuiza (2018) manifiesta que el trabajador es objeto de protección especial del Estado, porque siento la parte más débil de la relación contractual, necesita de un contrapeso protector, un orden legal, que contribuya a nivelar sus condiciones de inferioridad frente al empleador.

Ahora bien, los sueldos y salarios pueden estipularse libremente entre empleador y trabajador, respetando siempre el mínimo legal que comprende la remuneración básica unificada, remuneraciones que dependen del giro ordinario del negocio del 
empleador quien por lo general será el encargado de establecer el estipendio, a las cuales se adhiere el trabajador a fin de acceder a la relación laboral. Para, Bio Gaidolfi, (2017):

La remuneración no solamente debe cubrir las propias necesidades del trabajador sino también las de su familia, así como proporcionarle bienestar tanto material como espiritual ya que a través del trabajo no solamente se satisfacen necesidades materiales, sino que el trabajador también procura para si el bienestar espiritual, toda vez que es justamente a través del trabajo que puede desarrollar su proyecto de vida. (p.139).

En tal sentido, la remuneración es la contraprestación que percibe un trabajador por dar o brindar sus servicios profesionales u ocupaciones. Según Ccaulla Flores (2018) la remuneración es aquel ingreso económico producto de la prestación de un servicio a favor de una organización, la cual varia en funciones a los cargos que el trabajador pueda ostentar.

Sueldo

- Estipendio que el empleador paga al empleado

- Se paga por meses, incluidos los días no laborables

- El plazo para el pago no puede exceder de un mes

Salario

- $\quad$ Estipendio que paga el empleador al obrero

- $\quad$ Se paga por jornadas de labor (jornal), por unidades de obra o por tarcas

- El plazo para el pago no puede ser mayor de una semana.

Tipos de remuneración variable

- Bonos de permanencia;

- Bono por terminación del proyecto;

Incentivos:

- Participación en beneficios;

- $\quad$ Participación en desempeño;

- Basado en desempeño individual.

Reconocimiento:

- $\quad$ Concesión de premios;

- Reconocimiento de la Dirección 
- $\quad$ Nominaciones. (Hidalgo y Jaya, 2015).

Cuando la persona trabajadora renuncia, presenta desahucio o es despedida del trabajo, tiene derecho a recibir la bonificación e indemnización, que establecen los artículos 185 y 188 del Código del Trabajo; valores que serán calculados con la última remuneración percibida; normas jurídicas que afectan el derecho económico de quienes han recibido remuneración mixta o variable, pues no siempre la actual es la mejor y en ocasiones el empleador previo a despedirle, le envía de vacaciones, evita que tenga comisiones, etc.; a fin de pagar una menor indemnización.

El elemento diferenciador de las remuneraciones variables por su naturaleza misma es el rendimiento, Lautaro Fariña (2015) menciona la diferencia de la remuneración variables es que el resultado mensual total no es constante entre uno y otro mes, habiéndose producido contantes confusiones, calificándose como remuneraciones fijas o por arrojar simplemente diversos resultados de un mes a otro; otras dependientes únicamente del rendimiento del trabajador que han sido calificadas como eventuales, por el hecho de pactarse con una periodo superior al mes.

Por otro lado, se entiende por comisión el beneficio económico, proporcional a la cuantía de los negocios con el cual se retribuye al trabajador o comisionista según el resultado obtenido en la gestión u operación en que haya intervenido en nombre y por cuenta del empresario o comitente. Es una forma de participación en los ingresos, utilidades o ventas de una empresa a la que se pertenece activamente (Cabanellas, 2018). La remuneración por comisión difiere esencialmente en relación a los sujetos que participan de esta forma de ser remunerados. Hay determinadas actividades como las de los viajantes, corredores, cobradores, repartidores y otros trabajadores, en que la retribución es proporcional al valor de los negocios logrados por el agente para la empresa a los beneficios obtenidos con la intervención del trabajador.

Es importante destacar que la Constitución, en el artículo 328 define qué se considera remuneración para el pago de indemnizaciones, pero no indica que el cálculo deba hacerse con la última remuneración, pues es la ley que regula esta situación, como se indicó antes. 
Las liquidaciones que se realizan a fin de determinar el valor correspondiente a indemnización por despido intempestivo, por desahucio y decimotercera remuneración, serán realizados en base a la remuneración que percibía el trabajador al momento del despido o terminación del contrato, por lo que en las circunstancias actuales, tomando en cuenta la recesión global, se verían dilapidadas las indemnizaciones que por ley corresponden, ya que la base utilizada para la liquidación corresponde a una realidad económica distinta a la que usualmente acontecía, por tanto, la normativa aplicada a la situación conlleva a un desmedro de los derechos que corresponden como trabajador, en negocio que ha tenido más tiempo en actividad que en inactividad a causa de la pandemia y las medidas de control.

Se plantea como objetivo general analizar jurídicamente la Incidencia de la última remuneración en la liquidación por terminación del contrato de trabajo en Ecuador.

\section{METODOLOGÍA}

La metodología utilizada desde la perspectiva cualitativa, se apoyó con el método de investigación el analítico-sintético, ya que mediante el análisis que se realizó se descompuso el tema investigado en sus partes y cualidades más relevantes; por otro lado, con la síntesis se estableció la unión entre todas las partes estudiadas del tema investigado, de los hechos con el mecanismo de generalización del actuar que ha sido apreciado (Behar, 2008). Asimismo, se utilizó el análisis documental con la finalidad de adquirir información sobre los derechos laborales que, a lo largo del tiempo en Ecuador, lo cual para eso se investigó en el Códigos de Trabajo y libros relacionados con el Derecho Laboral referidos en la bibliografía, artículos de revista, normativa, y sobre todo jurisprudencia; produciéndose así aportes al derecho en la institución del debido proceso en la garantía del deber de motivar de las autoridades judiciales (Bravo, Narváez, Vázquez y Erazo, 2020). Se realizaron entrevistas a abogados conocedores de la materia laboral y a jueces laborales en la ciudad de Santo Domingo para que desde su experiencia manifiesten como en las indemnizaciones de trabajadores con remuneraciones con ingresos variables pueden causar vulneraciones al derecho al trabajo reconocido en la Constitución. 


\section{RESULTADOS}

Se presentan el resultado de las entrevistas formuladas a informantes clave, con experiencia en el tema de investigación.

\section{Cuadro 1.}

Entrevistas

\begin{tabular}{|c|c|}
\hline Entrevista $\mathrm{N}^{\circ} 1$ & 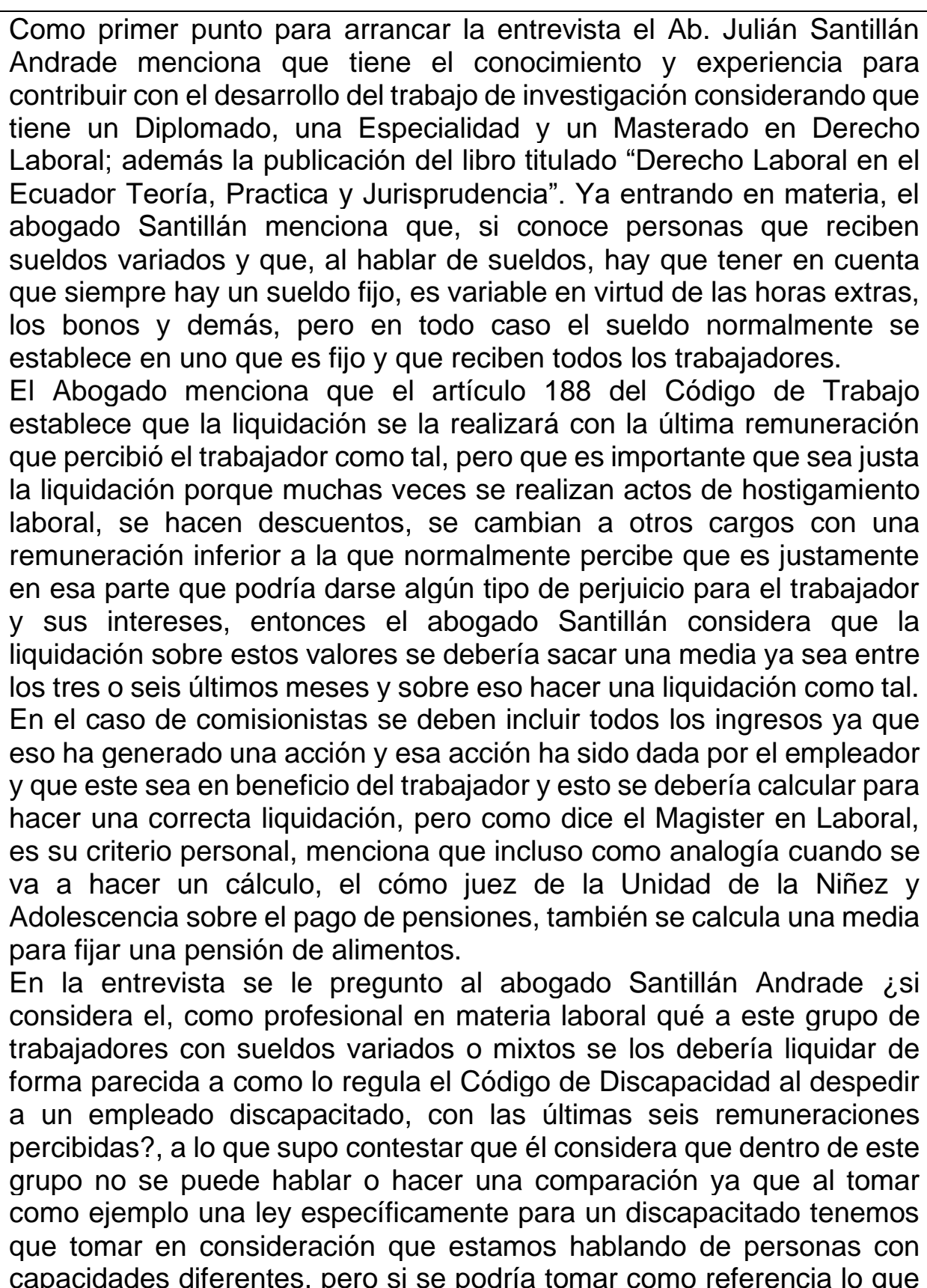 \\
\hline
\end{tabular}




\begin{tabular}{|c|c|}
\hline & $\begin{array}{l}\text { indica este Código para una correcta liquidación hacia este tipo de } \\
\text { trabajadores con sueldos variados y su terminación laboral tanto como } \\
\text { despido intempestivo o una renuncia voluntaria. } \\
\text { Finalmente el Abogado menciona que personalmente considera que no } \\
\text { existe vacío legal sobre la liquidación de un trabajador con la última } \\
\text { remuneración, sino que más bien resulta injusto, pero vacío no hay } \\
\text { porque si está establecido dentro de la Ley que sea la última } \\
\text { remuneración de un trabajador, pero dentro de la práctica diaria al } \\
\text { momento de hacerse el desahucio o demandarse por un requerimiento } \\
\text { de despido intempestivo con un valor, ahí es que se da realmente el } \\
\text { perjuicio al trabajador en alguno casos, porque se toma de base la última } \\
\text { remuneración y esta puede ser minina en relación a otras } \\
\text { remuneraciones, entonces no se estaría hablando de un vacío legal sino } \\
\text { de una injusticia al momento de realizar un cálculo al sacar el valor de la } \\
\text { última remuneración al ser despedido o poner una renuncia voluntaria, } \\
\text { pero para finalizar la entrevista hay que recordar que lo justo no siempre } \\
\text { es lo legal y lo legal no siempre es lo justo. }\end{array}$ \\
\hline Entrevista $\mathrm{N}^{\circ} 2$ & $\begin{array}{l}\text { Como primer punto para arrancar la entrevista el entrevistado Ab. Víctor } \\
\text { Hugo Moya menciona que tiene el conocimiento y experiencia para } \\
\text { contribuir con el desarrollo del trabajo de investigación considerando que } \\
\text { viene desarrollando alrededor de siete años en la docencia universitaria } \\
\text { en el campo labora, e inclusive en la práctica también en el libre ejercicio } \\
\text { del derecho se encuentra dedicado al ámbito de defensa de los derechos } \\
\text { del trabajador. } \\
\text { Ya entrando en materia el entrevistado menciona que quizás el } \\
\text { tecnicismo de establecer remuneraciones variables se da por el mismo } \\
\text { desarrollo y avance de la tecnología, de la ciencia, del desarrollo porque } \\
\text { una remuneración variable viene a considerarse una información falsa } \\
\text { ante las instituciones legalmente creadas por el legislador o por el } \\
\text { Estado para el control de los derechos de los trabajadores como es por } \\
\text { ejemplo el IESS, entonces allí hay una falsedad de información por una } \\
\text { cosa se informa al IESS o Ministerio de Relaciones Laborales y otra es } \\
\text { la realidad económica del trabajador entonces, el abogado Moya } \\
\text { considera que existe una falta de control o de legislación que pueda } \\
\text { controlar esa diferencia entre remuneración fijada como comisiones u } \\
\text { otros ingresos que tenga un trabajador. } \\
\text { El entrevista establece que el artículo } 188 \text { de la materia que se hace } \\
\text { referencia en la investigación que es laboral, hace notar el despido } \\
\text { intempestivo y para ello pues una de sus formas para el cálculo nos dice } \\
\text { que se tomará como base la última remuneración, el abogado Moya } \\
\text { considera que sería muy prudente establecer las remuneraciones de los } \\
\text { últimos doce meses, se opone desde su punto de vista a que sea de más } \\
\text { tiempo por cuanto año a año cambia el salario básico e inclusive pues a } \\
\text { veces también hay una alza en las remuneraciones y podríamos } \\
\text { extender a un máximo de mayor tiempo y perjudicar los derechos del } \\
\text { trabajador y de esta manera evitar alguna maniobra que puedan aplicar } \\
\text { los empleadores. } \\
\text { Por otro lado, en cuanto a si considera que se debería liquidar con las } \\
\text { últimas seis remuneraciones como es el caso de las personas con }\end{array}$ \\
\hline
\end{tabular}




\begin{tabular}{|c|c|}
\hline & $\begin{array}{l}\text { capacidades diferentes, menciona que no, ya que hay que tomar en } \\
\text { cuenta que la norma de las capacidades especiales es una norma } \\
\text { especial y no podemos mezclar una persona con capacidades } \\
\text { especiales con otro ciudadano común que si esta con las condiciones } \\
\text { físicas mentales para desarrollar una actividad normal, entonces para mi } \\
\text { debe existir siempre una diferenciación entre las dos personas, pero } \\
\text { efectivamente se debería considerar una media en donde se pueda } \\
\text { establecer una justa liquidación tomando en cuenta que este grupo de } \\
\text { trabajadores no siempre ganan un mismo sueldo sumado a varios rubros } \\
\text { que en determinado mes puede ser inferior y de esta manera el } \\
\text { empleador podría aprovechar para deshacerse por cualquiera que fuera } \\
\text { las razones de un trabajador; así como también; el trabajador podría } \\
\text { buscar su renuncia cuando este sueldo sea mucho mejor. } \\
\text { Además, menciona que definitivamente como profesional en la rama } \\
\text { laboral considera que no podemos hablar de vacíos legales porque } \\
\text { efectivamente nuestro Código de Trabajo si indica cómo se debe liquidar } \\
\text { a un trabajador en los casos que ya conocemos que se aplique la } \\
\text { terminación de una contrato tanto como el empleador y el trabajador, } \\
\text { pero en ocasiones si podríamos ver que no existe una proporcionalidad } \\
\text { justa ya q en este grupo de trabajadores pueden tener un ingreso muy } \\
\text { bueno así como también muy malo. } \\
\text { Finalmente, reza que en honor a la verdad ha desahuciado en los últimos } \\
\text { años inclusive ha sido abogado de los empleadores, ha plateado } \\
\text { desahucios, visto bueno. Esto ha conllevado de una u otra manera que } \\
\text { uno pues ejerce el derecho de quien le contrata, el entrevistado es muy } \\
\text { honesto en decir la verdad ya que dice que como profesional del derecho } \\
\text { tienes que defender a quien te paga. Por último, señala que son muy } \\
\text { pocos los desahucios en los últimos años pues prácticamente podríamos } \\
\text { decir que el transcurso del año unos diez casos aproximadamente de los } \\
\text { cuales en unos se ha podido justificar el desahucio otros se han llegado } \\
\text { a una conciliación y otros no se ha logrado mayor cosa. }\end{array}$ \\
\hline Entrevista $\mathrm{N}^{\circ} 3$ & $\begin{array}{l}\text { El entrevistado es juez de la Unidad Judicial Laboral de Santo Domingo, } \\
\text { Ab. Mgs. José Luis Maldonado Cando, el cual manifiesta que tiene } 15 \\
\text { años de experiencia en el libre ejercicio, casi } 3 \text { años como Director } \\
\text { Provincial del Ministerio de Relaciones Laborales, en ese entonces } \\
\text { Ministerio de Trabajo y actualmente lleva ejerciendo el cargo de Juez de } \\
\text { la Unidad Judicial Civil, Mercantil y Laboral en Santo Domingo por } \\
\text { aproximadamente } 6 \text { años. } \\
\text { El entrevistado manifiesta que efectivamente tiene claro lo que es el } \\
\text { sueldo variado, establece que comprenden las comisiones u otros rubros } \\
\text { que podría tener cualquier trabajador que labore bajo esta modalidad en } \\
\text { una relación de dependencia. Por otro lado, el entrevistado sostiene que } \\
\text { no conoce específicamente sobre cuántos casos por desahucio de } \\
\text { trabajadores con ingresos variables ingresa, pero lo que si puede decir } \\
\text { con certeza es que en su unidad judicial conocen sobre actas de finiquito } \\
\text { que adjuntan a los procesos judiciales en las cuales se puede apreciar } \\
\text { de que consta un rubro por desahucio en el cual el trabajador lo recibe } \\
\text { por efectos de terminación de la relación laboral, ya sea porque el mismo } \\
\text { trabajador presento su solicitud de desahucio al patrono o porque }\end{array}$ \\
\hline
\end{tabular}




\begin{abstract}
terminó la relación laboral por acuerdo de las partes, sin embargo la ley otorga este rubro a favor del trabajador, frente a esta situación hay que tomar en cuenta que el desahucio dependerá mucho de la remuneración que el trabajador haya estado recibiendo al tiempo de terminarse la relación de trabajo.

El entrevistado manifiesta que bajo su criterio, el empleador debe calcular y tomar en consideración la última remuneración tomando en cuenta el Art. 95 inciso primero del Código de Trabajo, ya depende del criterio que tenga en su cabeza el despedirlo y el último mes no lo hace trabajar absolutamente nada y frente a eso lo despide para que tome esa remuneración y en consecuencia de aquello pague menos la indemnización por despido intempestivo siempre y cuando el empleador ya lo tenga premeditado, mientras no el trabajador tiene derecho a recibir su indemnización tomando en cuenta los componentes del Art. 95 del Código de Trabajo.

El Dr. José Luis sostiene que si es que no le pagan al trabajador en base a la ley estarían siendo sus derechos vulnerados porque existe norma expresa, la ley manda, prohíbe o permite, por lo tanto ordena al empleador que se liquide como indica claramente en su norma que sería la última remuneración; pero, es ahí donde el empleador debe justificar en caso de que realice con uno de sus trabajadores un perjuicio realizando de forma injustificada su baja de sueldo para así indemnizar al trabajador con un sueldo más bajo del puede haber recibido anteriormente.

Finalmente, el entrevistado manifiesta que de existir malas interpretaciones por el empleador, existir algún vacío legal o al existir alguna duda, lo importante es clarificar o llenar estos vacíos a fin de que no salgan perjudicado absolutamente nadie, en este caso ni el trabajador ni el empleador. Frente a estas dudas, a estos vacíos se debe tomar en cuenta que para eso está la investigación para eso están las universidades, justamente a través de los diferentes programas de titulación que se presenten propuestas a fin de que los derechos de los trabajadores, así como los derechos de todas las personas de alguna forma sean compensados, no sean violentados, ni vulnerados por una mala interpretación por el empleador o por cierto abogados que solo buscan de alguna manera inclinar la balanza a favor del empleador y no a favor de las justicia.
\end{abstract}

Fuente: Entrevistas

\title{
DISCUSIÓN
}

En el país no se reconocen los derechos de una manera justa a los trabajadores que perciben remuneraciones variables. Teniendo en cuenta que la remuneración variable constituye todos los elementos que contiene una remuneración fija, y se hace efectiva a raíz de los logros individuales o por el desempeño o crecimiento de la empresa; se 
puede decir que la aplicación de la remuneración variable cambia el pensamiento de los trabajadores, lo cual puede llegar a potenciar sus habilidades, las que pueden cuantificarse en sus ingresos.

En razón de lo mencionado se puede traer a la presente investigación los tipos de remuneración variable, como es la evaluación de desempeño del trabajador, ya que en cuando existe una remuneración variable es de suma importancia la aplicación de la evaluación del desempeño para analizar las contribuciones que los empleados pueden entregar a una empresa.

Es decir, el proceso debería estar ajustado a los resultados que el trabajador tenga, por tal razón es considerable que la indemnización de un trabajador con remuneraciones variables sea justa, y como decían los entrevistados, que se haga una media para poder realizar una indemnización acorde a las funciones y desempeño que alguna vez el trabajador mostró dentro de la empresa.

Por otro lado, en el país la remuneración salarial de los trabajadores en el sector privado se ha convertido en un problema de dificultad alta de tratar y armonizar a lo largo de los años, cabe mencionar que dentro de la presente investigación en materia laboral se planteó las ventajas y desventajas de la remuneración variable como sistema de retribución o remuneración.

Se ha podido establecer que el salario es variable cuando la remuneración no se paga en función de un periodo de tiempo, sino en función de lo que el trabajador hace, el típico ejemplo de lo ya mencionado es que un salario variable es el pago de comisiones, pues el salario dependerá de las comisiones que gane el trabajador en un periodo determinado.

Queda esclarecido que los salarios variables pueden ser percibidos de dos maneras:

- $\quad$ En forma de comisión

- $\quad$ En forma de complemento que depende de los resultados que obtenga la organización. 


\section{CONCLUSIONES}

Se puede concluir que, si existe incidencia en la última remuneración de un trabajador puesto que los sueldos variados o mixtos tienen ventajas, pero también tienen desventajas, ya que, así como puede que mensualmente destaquen por su desempeño en el trabajo, puede que otros menos no reciban más que el mínimo porque su desempeño dentro de la empresa no cumplió las expectativas ese mes, por tal razón se puede entender que es un arma de doble filo.

Al existir diferentes sueldos al momento de establecer una relación laboral debemos tomar en cuenta que si existe un empleado en el cual fue contratado con sueldo determinado y hay otro grupo de trabajadores el cual su sueldo variará del acuerdo que tomen entre las partes en donde su remuneración dependerá del giro del negocio en el que su sueldo va a ser diferente cada mes.

Cabe mencionar que además esto de que se liquide en base a la última remuneración puede ser algo de que se beneficie la empresa, ya que la empresa puede cesar las funciones de un trabajador cuando haya tenido un rendimiento muy bajo, por lo que su liquidación debería ser baja, pero así mismo el empleado podría poner la renuncia cuando haya ganado una última remuneración que sea alta.

Finalmente, no se puede hablar de que exista una vulneración, aunque si podría existir una mala aplicación a favor del empleador, ya que puede utilizar situaciones donde se pueda perjudicar a este sector de trabajadores que tienen ingresos diferentes meses a mes.

\section{FINANCIAMIENTO}

No monetario.

\section{AGRADECIMIENTO}

A la Universidad Regional Autónoma de los Andes, Santo Domingo, por motivar el desarrollo de la Investigación. 


\section{REFERENCIAS CONSULTADAS}

Asamblea Nacional Constituyente de la República del Ecuador, (2008). Constitución de la República del Ecuador. [Constitution of the Republic of Ecuador].Montecristi. Registro Oficial 449 de 20-oct-2008. Recuperado de https://n9.cl/sia

Behar Rivero, D. (2018). Metodología de la Investigación. [Investigation methodology]. Recuperado de https://n9.cl/k9q2

Bio Gaidolfi, C. (2017). Trabajo, empleo y remuneración justa: análisis comparativo de la encíclica "laborem exercens" y la legislación laboral peruana. [ Work, employment and fair remuneration: comparative analysis of the encyclical "laborem exercens" and Peruvian labor legislation]. Universidad Católica Sedes Sapientiae. Lima. Recuperado de: https://n9.cl/fstvv

Bravo Núñez, A. d., Narváez Zurita, C. I., Vázquez Calle, J. L., y Erazo Álvarez, J. C. (2020). Reparación integral de la Corte Interamericana de Derechos Humanos: Sentencias de acción extraordinaria de protección. [ Comprehensive Reparation of the Inter-American Court of Human Rights: Judgments of Extraordinary Action for Protection]. Iustitia Socialis, 5(8),584-602. http://dx.doi.org/10.35381/racii.v5i8.592

Cabanellas, G. (2018). Diccionario Enciclopédico de Derecho Usual. [ Encyclopedic Dictionary of Usual Law]. Buenos Aires: Heliasta. Recuperado de: https://n9.cl/7lwu

Ccaulla Flores, L. (2018). La remuneración salarial y el desempeño laboral en la Unidad de Gestión Educativa Local № 06 - Ate, 2018. [ Salary remuneration and work performance in the Local Educational Management Unit No. 06 - Ate, 2018]. TESIS. Escuela de Posgrado. Universidad Cesar Vallejo. Recuperado de: https://n9.cl/ckf5f

Congreso Nacional. (2005). Código del Trabajo. [ Work code]. Codificación 17 Registro Oficial Suplemento 167 de 16-dic.-2005. Recuperado de: https://n9.cl/652cf

Hidalgo Sanguano, M. y Jaya Álvarez, K. (2015). Análisis de la remuneración variable como herramienta de la gestión estratégica. [ Analysis of variable remuneration as a tool for strategic management]. Tesis de Maestría. Universidad de las Fuerzas Armadas. Recuperado de: https://n9.cl/p1ck2 
Lautaro Fariña, Q. (2015) Remuneración Variable: Elemento diferenciador y relación con las remuneraciones participativas. [Variable Remuneration: Differentiator Element And Relationship With Participating Remunera Ti On]. Revista Chilena de derecho del Trabajo y de la Seguridad Social, 6(11),93-105. Recuperado de: https://n9.cl/wkuew

Masabanda Analuiza, G. (2019). Instrumentos Jurídicos Aplicables en la Contratación Individual de Trabajo. [Legal Instruments Applicable in Individual Labor Contracting]. Publicaciones y Libros - Consejo Editorial Uta, O(1), 148. Recuperado de: https://n9.cl/pkhcw 